\title{
Figure del disamore. I ritratti e le imprese di Bussy- Rabutin
}

Daniele Santero

\section{(2) OpenEdition}

1 Journals

\section{Edizione digitale}

URL: http://journals.openedition.org/studifrancesi/9761

DOI: 10.4000/studifrancesi.9761

ISSN: 2421-5856

\section{Editore}

Rosenberg \& Sellier

\section{Edizione cartacea}

Data di pubblicazione: 1 août 2017

Paginazione: 223-234

ISSN: 0039-2944

\section{Notizia bibliografica digitale}

Daniele Santero, «Figure del disamore. I ritratti e le imprese di Bussy-Rabutin», Studi Francesi [Online] 182 (LXI | II) | 2017, online dal 01 août 2018, consultato il 06 janvier 2021. URL: http:// journals.openedition.org/studifrancesi/9761 ; DOI: https://doi.org/10.4000/studifrancesi.9761

\section{cc) (1) $\odot$}

Studi Francesi è distribuita con Licenza Creative Commons Attribuzione - Non commerciale - Non opere derivate 4.0 Internazionale. 


\section{Figure del disamore. I ritratti e le imprese di Bussy-Rabutin}

\section{Abstract}

Even more than in his Mémoires, Bussy-Rabutin gives the summary of his life and loves by collecting in his castle portraits and devises during the exile. The essay focuses on this figurative autobiography, paying attention to contemporary symbolic theory.

Il 10 settembre 1666 Roger de Bussy-Rabutin scende dalla sua carrozza nella sua piccola corte, dopo quattro giorni di viaggio da Parigi. In primavera ha soggiornato per la seconda volta ${ }^{1}$ alla Bastiglia; ne è uscito gravemente malato e dal suo re ha ricevuto la condanna all'esilio nelle forme di un consiglio amorevole: «pour recouvrer entièrement votre santé, il serait nécessaire que vous poussiez aller prendre l'air chez vous en Bourgogne» ${ }^{2}$. La parola del re sancisce la sventura definitiva, conquistata ufficialmente con la diffusione del manoscritto della Histoire amoureuse des Gaules; e nello stesso tempo si rivela efficace: all'arrivo in Borgogna, scrive alla fine dei Mémoires, il corpo è già più rinfrancato dalla salubrità dell'aria che abbattuto dalla fatica del viaggio. Ma se si deve trovare un punto in cui lo spirito si sia indebolito quasi fino a spegnersi e a svanire del tutto, si può fissare quella prima sosta, la prima passeggiata negli appartamenti vuoti della sua residenza: nelle sale silenziose Bussy deve aver intuito, con un certo spavento, il vero senso della retraite e di una vita contemplativa sconosciuta nella conversazione dei salotti, come nel fragore degli assalti e delle battaglie.

Rispetto a quello dell'bonnête homme che avrebbe voluto diventare, l'esprit di Bussy è sempre stato più incline al brio e alla giocosità del galant bomme che alle qualità più profonde dell'bonnêteté: in realtà, se si fosse limitato all'esercizio della più frivola galanteria, effimera «comme une fleur ou comme une songe» ${ }^{3}$, non sarebbe finito in quelle stanze desolate, dopo aver lasciato nel mondo la fama, meritata ma anche eccessiva, di empio e libertino.

(1) Nel 1641 Bussy è comandante di un reggimento di cavalleria di guarnigione a Moulins: allontanatosi dal campo per alcuni giorni, il conte è giudicato responsabile del contrabbando di sale avviato dai suoi soldati. In prigione ha modo di frequentare il maresciallo di Bassompierre, nemico di Richelieu: «fut un de ceux qui deplaisoit le plus au Cardinal: sa familiarité auprés du Roi, sa liberté de parler [...] le firent craindre \& hair de ce Ministre» (Discours du comte de Bussy-Rabutin à ses enfants sur le bon usage des adversités et les divers événements de sa vie, Paris, Anisson, 1694, pp. 159-160). Per un'introduzione alla biografia di Bussy-Rabutin si vedano in particolare: J. OrIEux, Bussy-Rabutin. Le libertin galant homme 1618-1693, Paris, Club des Éditeurs, 1958; J. DuchênE, Bussy-Rabutin, Paris, Fayard, 1992; D.H. VINCENT, Bussy-Rabutin. Le libertin puni, Paris, Perrin, 2011.

(2) Comte de Bussy-Rabutin, Mémoires, édition présentée et annotée par D.H. Vincent, Paris, Mercure de France, 2012, p. 337. Apparsi postumi nel 1696, i Mémoires sono stati pubblicati per la prima volta in due volumi nel 1857 (Mémoires de Roger de Rabutin, comte de Bussy, avec une préface, des notes et des tables par L. Lalanne, Paris, Charpentier, 1857). Seguendo un approccio ammesso dallo stesso Bussy, vengono escluse dall'edizione le lettere di servizio, le descrizioni degli assedi e delle formazioni di battaglia.

(3) A. Gombaud, Chevalier de Méré, Première conversation, in ID., Euvres de Monsieur Le Chevalier de Méré, Amsterdam, Mortier, 1692, vol. I, p. 199. 
A tutela di chi desiderava piacere a corte, il cavaliere de Méré suggeriva la sostanziale identità tra esprit e jugement, accessorio della saggezza, necessario a valutare le circostanze, «à les savoir considerer à toutes sortes d'égards, à juger nettement de ce qu'elles sont»e, soprattutto, «à sçavoir prendre les bonnes voyes» ${ }^{4}$. Poco prima di conoscere Bussy, negli Entretiens Bouhours aggiungeva alle stesse conclusioni la chiave di una ingegnosa rappresentazione simbolica: «inseparable du bon sens», il bel esprit «est de la nature de ces pierres précieuses, qui n'ont pas moins de solidité que d'éclat», «un corps solide qui brille» ${ }^{5}$. Ma Bussy si rivolge tardi ai consigli dei moralisti mondani; soprattutto, anche se nelle lettere filosofeggia sulla mediocritas oraziana e nel suo salone si fa dipingere un diamante con il motto «Plus de solidité que d'éclat», lo fa, in fondo, per trovarsi al centro di una nuova scena e prolungare la celebrazione di una qualité percepita come vocazione («Lorsque j’entrai dans le monde, ma première et ma plus forte inclination fut de devenir honnête homme» ${ }^{6}$ ). Dopo, Bussy-Rabutin si voterà al fallimento ogni volta che sta per diventare ciò che vorrebbe, un uomo distinto nelle armi e nella qualità, per una qualche leggerezza dello spirito superiore alla misura.

Così, dopo la famosa débauche di Roissy, dove con altri galantuomini avrebbe tenuto a battesimo un porcellino da latte nella Pasqua del 1659, la Regina stessa non può che riconoscergli il dono dell'esprit come si riconosce un difetto: «Oh! Pour de l'esprit, Bussy, reprit la Reine, vous en avez beaucoup ${ }^{7}$. Bussy tenterà di ristabilire la giusta misura, ma la sovrana lo ha rivelato. Per lui vale lo stesso principio scandito da Bouhours nella sua apologia; l'esprit è una forza che si afferma nelle azioni, pressoché irresistibile: «il va toûjours droit au but, en quelque matière que ce soit, sans s'écarter, ni sans s'amuser en chemin» ${ }^{8}$. Se può vacillare quando viene estromesso dal mondo, alla fine il suo barlume sopravvive anche nella pura desolazione dell'esilio, scrive Bussy alla Montpensier, «dans la disgrâce du roi, c'est-à-dire dans l'enfer de ce monde»?.

Bussy cade su uno dei principi più ribaditi dell'intero savoir-vivre, sottinteso alla battuta della sua regina. L'arte di piacere si fonda anche su una certa economia dell'esprit e su una finzione di «conformità» in cui Méré rintracciava ancora una forma di cortesia e la cura di una reciprocità del piacere stesso. Quando a Mardyck si lancia all'assalto di una trincea spagnola, sotto gli occhi del Grand Condé, quando rapisce Madame de Miramion o alla Bastiglia risponde sprezzante a chi lo interroga («si tous ceux qui valent moins que moi étaient à la Bastille, il y aurait peu de gens de reste

(4) Ivi, p. 10.

(5) D. Bounours, Les entretiens d'Ariste et d'Eugène, édition établie et commentée par B. Beugnot et G. Declercq, Paris, Champion, 2003, p. 239. Entrato in contatto con Bussy subito dopo l'uscita degli Entretiens (1671), il padre gesuita diventa presto uno dei corrispondenti più cari al conte, che non a caso lo sceglie come primo lettore dei Mémoires autografi (1673). Alla morte di Bussy, con cui intrattiene una fitta corrispondenza (Bussy-Rabutin, Correspondance avec le Père Boubours, introduction et notes par C. Rouben, Paris, Nizet, 1986), Bouhours curerà l'edizione postuma degli stessi Mémoires (1696).

(6) Comte de Bussy-Rabutin, Mémoires cit., p. 31.

(7) Ivi, p. 250.

(8) D. BouHours, Les entretiens cit., p. 241.

(9) Anche la prima edizione dell'epistolario di Bussy viene affidata alle cure di Bouhours e pubblicata in quattro volumi nel 1697. L'edizione più affidabile e completa delle lettere, tenuta come riferimento per questo saggio, è curata da Lalanne tra il 1858 e il 1859 (Correspondance de Roger de Rabutin, comte de Bussy avec sa famille et ses amis, avec une préface, des notes et des tables par L. Lalanne, Paris, Charpentier, 1858 , vol. II, p. 327). Per un quadro generale dell'epistolografia francese contemporanea a Bussy, ricco di riferimenti all'opera del conte, si veda B. BRAY, Épistoliers de l'âge classique: l'art de la correspondance chez Madame de Sévigné et quelques prédécesseurs, contemparains et béritiers, études revues, réunies et présentées avec la collaboration de O. Richard-Pauchet, Tübingen, Gunter Narr Verlag, 2007. 
pour les interroger» ${ }^{10}$ ), il conte persegue sempre lo stesso scopo: vivere eroicamente, guadagnarsi un éclat, assecondare a qualsiasi costo il moto volubile dell'amor proprio. Lo stesso esilio sarebbe l'occasione perfetta per un disarmo della propria individualità, una retraite dello spirito che si defila dalla scena mondana, scortato dal disprezzo del monde della malinconica Madame de Scudéry e dai libri di meditazione ricevuti dagli amici Rapin e Bouhours. Ma l'ultima lettera scritta a Madame de Sévigné, nel dicembre del 1692, lo trova ancora alle prese, più che settantenne, con una certa verve spirituale: «Il faut que j'amuse ancore mon esprit» scrive alla cugina, a suo tempo colpita con il perfido ritratto dell'Histoire ${ }^{11}$. Quanto alla permanenza dell'esprit nell'«inferno del mondo», tutto appare già chiaro nel bilancio comunicato a Bouhours dopo otto anni d'esilio. Dopotutto, Bussy non si è mai mosso dal centro della scena. Lo spirito trionfa, alla fine, sulla stessa fortuna: «La Fortune est une sotte, quand elle a cru m'avoir fait le plus grand mal du monde» ${ }^{12}$.

Iniziati durante la reclusione alla Bastiglia, i Mémoires sono il luogo privilegiato in cui portare avanti la rappresentazione minuta di una qualità che forse non sarebbe nulla, proprio come in una conversazione tra bonnêtes hommes, senza la possibilità di brillare e il plauso degli interlocutori. D'altra parte, la necessità di chiarire la verità di «cose degne di essere scritte» ${ }^{13}$, che in Casanova veniva dopo la scrittura di pagine «degne di essere lette», capaci di restituire «una "doppia" vita nella scrittura autobiografica» ${ }^{14}$, è il vero spunto dei Mémoires e nello stesso tempo il loro fine. All'inizio della sua storia, dove Casanova, uomo dichiaratamente "non illustre", porrà una dichiarazione di ingenuità e inconsapevolezza necessaria per vivere ancora un po' nella sua pagina, Bussy pone un disegno da ripassare e precisare, un gesto eroico che non ha compiuto, ma per cui si può ancora spendere.

Strumento di conferma di una condotta più virtuosa delle apparenze, i Mémoires trattengono i loro scopi immediati. Si tratta, innanzitutto, di ripulire una reputazione compromessa a tal punto, dopo le plaisanteries dell'Histoire, da essere invocato come inventore di ogni maldicenza parigina («aussitôt qu'il y avait une raillerie malicieuse dans le monde on me l'attribuait» ${ }^{15}$ ). Soprattutto, la «grande air de verité» della sua autobiografia non poteva che essere un punto fermo per riguadagnarsi lo sguardo di Luigi. «Votre Majesté ne daignait me regarder; j'aime autant qu'elle me fasse mourir, Sire, si elle ne me regarde pas ${ }^{16}$ : prima di gettarsi in lacrime ai piedi del suo re, Bussy indica ciò che i suoi Mémoires dovranno attirare. Nella salle des devises c'è anche una meridiana colpita da un sole splendente in un cielo limpido: il motto, inventato secondo Bouhours dal conte di Croisi, recita «Si me mira me miran», se mi guarda mi

(10) Comte de Bussy-Rabutin, Mémoires cit., p. 306.

(11) Nel quadro dei rapporti tra la Sévigné e il cugino Bussy-Rabutin, turbati da screzi e litigi, sostenuti dal rabutinage, ovvero uno stile comunicativo giocoso e pungente, raffinatissimo e aperto ad ogni digressione, il ritratto letterario della donna inserito nell'Histoire amoureuse des Gaules rappresenta un episodio tra i più memorabili: «Madame de Sévigné è infida fino alle pupille degli occhi, fino alle palpebre: ha gli occhi di colori differenti, ed essendo lo specchio dell'anima, il fatto che siano diversi è quasi un avvertimento che la natura dà a chi l'avvicina, di non fare troppo affidamento sulla sua amicizia» (R. DE BUSSYRabutin, Storia amorosa delle Gallie, traduzione e note di R. Tinti, postfazione di D. Galateria, Palermo, Sellerio, 1992, p. 142).

(12) Correspondance de Roger de Rabutin cit., vol. II, p. 368

(13) G. Casanova, Storia della mia vita, a cura di P. Chiara e F. Roncoroni, Milano, Mondadori, 1983, vol. I, p. 6.

(14) G. Ficara, Casanova e la malinconia, Torino, Einaudi, 1999, p. 88. Sulla fortuna e i significati del genere autobiografico e memorialistico nel Seicento francese si vedano le conclusioni offerte da F. BRIOT, Usage du monde, usage de soi. Enquête sur les mémorialistes d'Ancien Régime, Paris, Seuil, 1994 e E. LESNE, La poétique des mémoires (1650-1685), Paris, Champion, 1996.

(15) Comte de Bussy-Rabutin, Mémoires cit., p. 290.

(16) Ivi, p. 294. 
guardano. Bussy è la meridiana, Luigi il sole che restituisce un senso alla sua esistenza: lo sguardo benevolo del re, invocato a salvare un uomo dal suo inferno, potrebbe anche raddrizzare una reputazione e restituirle l'ammirazione del mondo.

Se si escludono i Mémoires di Bassompierre, comunque criticati per la loro penuria documentaria, Bussy si ritrova privo di punti d'appoggio per la sua scrittura autobiografica, dal momento che, scrive nel 1670 alla Scudéry, le memorie dei grandi restituiscono ritratti di creature infallibili, «héros accomplis, qui n'ont jamais fait un faux pas» ${ }^{17}$. Al contrario, lo sguardo del re potrà essere riconquistato dalla confessione di tutti i suoi passi falsi anche perché questi sono innanzitutto divertenti. I Mémoires sono «quelque chose d'assez amusant» ${ }^{18}$ perché contengono anche le prime maldestre avventure amorose, i duelli, i dettagli delle débauches, la cronaca del rapimento della Miramion, i badinages in Catalogna con il Prince de Conti, sigillati da una Carte du pays de Braquerie che è la parodia della Carte du Tendre che Mademoiselle de Scudéry aveva appena allegato alla prima parte della sua Clélie (1654). Più radicalmente che in Bouhours, se non fosse anche incauto e gioioso il cammino dell'esprit nel mondo non avrebbe poi molto senso.

Anche più dei Mémoires, il luogo adibito alla libera rappresentazione di sé è un pannello o una parete delle stanze malandate del suo castello, bisognose di cure e di restauri. Avviati alla fine del 1666, i lavori nella residenza proseguono fino ai primi anni del decennio successivo, diretti in prima persona, affiancati da una fervente attività epistolare e dalla conclusione dei Mémoires. Quando sono ancora in corso Bussy assicura a Corbinelli, nel luglio del 1669, di stare allestendo una residenza inimitabile anche perché le sue pareti, non più spoglie, stanno diventando lo sfondo per le ingegnose combinazioni tra figura e parola che sono l'essenza di una devise ben riuscita.

Se le raccolte fondamentali di emblemi risalgono in Francia a un secolo prima e devono tutte qualcosa all'Alciato ${ }^{19}$, le riflessioni teoriche più significative sulla letteratura simbolica appaiono durante la giovinezza e la maturità di Bussy. La teoria si occupa per lo più di fissare regole chiare alla devise, ormai ridotta ad «ornement, jeu de cour, métaphore ingénieuse $\gg^{20}$, priva dell'aspirazione rinascimentale a sintetizzare e connettere $\mathrm{i}$ frammenti dell'universo visibile e invisibile. Legata alla fortuna dei geroglifici e fondata sulla stessa sacra alleanza tra le parole e le $\cos ^{21}$, la letteratura

(17) Correspondance de Roger de Rabutin cit., vol. I, p. 335.

(18) Ibidem.

(19) La prima traduzione della raccolta di Alciato, ad opera di Jean Le Fevre, è pubblicata a Parigi nel 1536, a cinque anni di distanza dalla prima edizione latina. Nello stesso anno vede la luce, in forma incompleta, la prima raccolta francese di emblemi, ad opera di Guillaume De La Perrière, riproposta in forma completa quattro anni dopo (Le Theatre des bons engins, auquel sont contenus cent Emblemes, Paris, Janot, 1540). Segue l'Hecatomgraphie di Corrozet (Paris, Janot, 1540), quindi, tra i testi più significativi, la Picta poesis di Barthélemy Aneau, subito tradotta in francese (Imagination poetique, traduicte en vers françois, des latins, E grecs, par l'auteur mesme d'iceux, Lyon, Bonhomme, 1552) e il tardo Pegme di Cousteau (Lyon, Bonhomme, 1555). Alla ricognizione testuale di Praz nel suo studio pioneristico sulla produzione simbolica del Seicento (M. PRAz, Studies in seventeenth century imagery, Roma, Edizioni di storia e letteratura, $1964^{2}$; prima: London, Warburg Institute, 1939) va ora affiancata la capillare rassegna offerta da A. ADAMS - S. RaWLES - A. SAunders, A bibliography of French emblem books of the Sixteenth and Seventeenth Centuries, Genève, Droz, 2002. Per un inquadramento critico delle opere e i significati della produzione simbolica si veda soprattutto: R.J. CLEMENTS, Picta poesis. Literary and bumanistic theory in Renaissance emblem books, Roma, Edizioni di storia e letteratura, 1960; A. SAUNDERS, The sixteenth-century french emblem book. A decorative and useful genre, Genève, Droz, 1988; EAD., The seventeenth-century french emblem. A study in diversity, Genève, Droz, 2000; Aspects of Renaissance and baroque symbol theory 1500-1700, edited by P.M. Daly and J. Manning, New York, AMS Press, 1999.

(20) A. SPICA, Symbolique bumaniste et emblématique. L'évolution et les genres (1580-1700), Paris, Champion, 1996, p. 439.

(21) Si veda, a proposito, la lettera indirizzata «aux bons espritz \& amateurs de lettres» da Corrozet in apertura dell'Hecamtographie: «Chascune hystoire est d'ymage illustrée | [...] Qu'on peult nommer lettre 
emblematica mirava a sanare la frattura tra sensibile e intelligibile, considerando il mondo come un liber naturae aperto a una lettura che ne sapesse decifrare lo spettacolo visibile e mettere ordine in un disordine apparente: «ce monde dont nous ne voyons que des fragments reste pour nous inconnaissable tant que les relations d'un fragment avec l'autre ne sont rétablies» ${ }^{22}$.

Quando invece deve chiarire l'origine delle imprese, Giovio non va oltre i «cimieri» e gli «ornamenti negli elmetti e negli scudi» dei guerrieri dell'Eneide o dei generali descritti da Stazio o Plutarco; ma non ha alcun dubbio, poi, nel collocare il punto d'avvio per la voga delle imprese nel «ricchissimo spettacolo» delle livree dei cavalieri francesi giunti con Carlo VIII in Italia (1494). L'idea di una modernità della devise si impone presto tanto nelle pagine degli accademici italiani quanto nella teoria francese; negli Entretiens di Bouhours, ad esempio, diventa uno dei tratti distintivi del genere: se gli emblemi sono vecchi quanto il mondo, «la Devise est nouvelle», «inconnuë aux temps des Héros» ${ }^{23}$.

Linguaggio vivo e produttivo, tutto teso a proclamare qualcosa di nobile sul proprio portatore («une action glorieuse, une passion honneste, une vertu», «quelque chose de grand, $\&$ d'illustre» ${ }^{24}$ ) più che a sondare occulte corrispondenze cosmiche, la devise si afferma nella corte di Francia anche per la frequenza e l'importanza del cerimoniale $^{25}$. In una pagina della Philosophie des images (1682) che Praz riprende per l'efficacia della sintesi, il gesuita Menestrier tratteggia un'intera corte alle prese con le invenzioni raffinate della moda simbolica: Monsieur Clément, sublime improvvisatore di motti ben noto allo stesso Bussy, lascia al figlio una raccolta di duecento pezzi «comme l'heritage de son esprit»; lo stesso Mazarino si misura nel gioco mondano, avendo portato con sé «ce goust \& cette inclination de son païs» ${ }^{26}$. Pronta a battagliare per il primato nell'invenzione dell'arte, la teoria francese riconosce il merito della perfezione agli accademici italiani e si forma soprattutto sui trattati e le raccolte di Giovio, Bargagli, Ruscelli, Ammirato e Ferro ${ }^{27}$.

hierogliphicque» (G. CORROZET, Hecamtographie cit., pagina non numerata). Per un'analisi dei significati del rapporto tra la letteratura emblematica e la riscoperta dei geroglifici si veda A. SPICA, Symbolique bumaniste et emblématique cit., pp. 45-90.

(22) Ivi, p. 169.

(23) D. BouHours, Les entretiens cit., p. 419. In realtà, soprattutto in ambito italiano, non pochi autori vollero stabilire un legame diretto tra geroglifici e imprese. Tasso dedica una larga parte del suo dialogo (Il Conte overo de l'imprese, 1594) a una discussione di questo presunto rapporto, finendo per «preferire una più neutra origine, analoga a quella postulata da Giovio» (G. Arbizzoni, «Un nodo di parole e di cose». Storia e fortuna delle imprese, Roma, Salerno, 2002, p. 81). Nel Cannocchiale Tesauro riconduce l'origine delle imprese al Cinquecento senza fare cenno alla discesa di Carlo VIII; i cavalieri non sono più francesi, ma sono già diventati campioni di «spiritose, frizzanti, \& erudite Argutezze» barocche (E. TESAURO, Il cannocchiale aristotelico o sia Idea dell'arguta et ingeniosa elocutione che serve a tutta l'Arte oratoria, lapidaria, et simbolica, Savigliano, Editrice Artistica Piemontese, 2000 [ristampa anastatica di: Torino, Zavatta, 1670], p. 626).

(24) D. Bouhours, Les entretiens cit., p. 365. Poco prima, per dare risalto e fondamento alla sua opinione, il gesuita aveva citato in francese e in italiano la diciannovesima tesi del Cannocchiale del «Comte Tesauro»: l'impresa è «Una metafora dipinta nello scudo de gli heroi» (ibidem, corsivo nel testo).

(25) La devise, conclude Chatelain, accompagna tutta la vita di un cortigiano, «dès rejouissances princières auxquelles il prend part en temps de guerre aux exploits qu'il accomplit en temps de guerre» (J. CHATELAIN, Livres d'emblèmes et de devises. Une anthologie (1531-1735), Paris, Klincksieck, 1993, p. 47). Per una panoramica antologica sul genere si rimanda anche a «Con parola brieve e con figura». Libri antichi di imprese e emblemi, introduzione di L. Bolzoni, Lucca, Fazzi, 2004.

(26) C.F. Menestrier, La Philosophie des images, composée d'un ample Recueil de Devises, E du Jugement de tous les Ouvrages qui ont êté faits sur cette matière, Paris, De La Caille, 1682, vol. I, pp. 105-107.

(27) Si tratta, a partire dal Dialogo del Giovio, dei testi di maggior successo in terra francese: S. BARGAGLI, Dell'imprese [...], Venezia, Francesco de' Franceschi, 1594; G. Ruscelli, Le imprese illustri. Aggiuntovi nuovamente il quarto libro da Vincenzo Ruscelli da Viterbo, Venezia, Francesco de' Franceschi, 1584; S. Ammirato, Il Rota overo dell'imprese, Napoli, Gio. Maria Scotto, 1562; G. Ferro, Teatro d'imprese, Venezia, Sarzina, 1623. Per una introduzione all'emblematica italiana si veda il recente The Italian Emblem. 
La convinzione comune a ogni autore di devises è che queste siano ben più di un'inezia tanto laboriosa da spopolare tra una folla di cortigiani annoiati. Se non può dissigillare il mondo delle parvenze, come l'emblema rinascimentale, l'impresa contiene comunque un po' di mistero: discende nelle profondità del mondo interiore e ne porta alla luce un dettaglio, senza liberarlo del tutto dalla sua oscurità naturale. Non sembra altro che «un jeu du Pinceau \& de la Plume», scrive Boissière, ma in realtà vuole «la chaleur d'une belle imagination», «la froideur d'un jugement profond», «un esprit vif \& brillant», «des finesses des langues» e «des secrets de la Philosophie» ${ }^{28}$. Il dominio assoluto della soggettività nello spazio della devise, tanto più illeggibile e oscura quanto più lontana dalla personalità del proprio autore, è ciò che la rende un codice congeniale all'egocentrico Bussy e nello stesso tempo la vera nota distintiva in seno alla letteratura simbolica ${ }^{29}$.

Bussy è attratto a tal punto dal potere espressivo delle combinazioni di parole e di cose da applicare la struttura della devise anche ai ritratti memoriali allineati nelle sue gallerie di hommes de guerre, rois de France, hommes illustres ${ }^{30}$. Di solito Bussy non va oltre una breve proposizione: dei grandi antichi e contemporanei riporta il nome, il titolo, la carica e un paio di qualità distintive o l'impresa più memorabile. Di ciascuno deve essere possibile conoscere quanto basta: sotto i volti le sue didascalie rispondono a uno scopo strettamente educativo che, come nei Mémoires, si intreccia con la ricerca di un duraturo amusement ${ }^{31}$ e deriva dai principi della pedagogia gesuita ${ }^{32}$.

Quando però il modello si trova al di fuori della grande storia e più vicino alla vicenda personale, Bussy esce allo scoperto: glossando i ritratti che le amiche gli inviano da Parigi tra il 1667 e il 1668 si libera tanto dello scrupolo dell'erudito quanto, di fronte agli antenati, della celebrazione della stirpe dei Rabutin, esaltata tra le devises con una fontana da cui sgorga un gran getto d'acqua con il motto «Altus ab origine alta» ${ }^{33}$. In effetti, per alcune sottoscrizioni Bussy deve avere provato lo stesso piacere di esercitare

A Collection of Essays, edited by D. Mansueto in collaboration with E.L. Calogero, Glasgow, Centre for emblem studies, 2007.

(28) Les Devises de Monsieur de Boissière, avec un Traitté des Regles de la Devise, par le mesme Autheur, Paris, Courbé, 1654, pagine non numerate. Quanto all'oscurità, Giovio aveva indicato la giusta misura tra le «cinque condizioni» dell'impresa perfetta: «non sia oscura di sorte ch'abbia mestiero della sibilla per interprete a volerla intendere, né tanto chiara ch'ogni plebeo l'intenda» (P. GIovio, Dialogo dell'imprese militari e amorose, a cura di M.L. Doglio, Roma, Bulzoni, 1978, p. 37).

(29) «Les paroles de l'Embleme peuvent dèmonstrer des choses universelles, \& tenir lieu de preceptes moraux, qui serviront aussi bien à tout le monde, qu'au propre Autheur de l'Embleme. Ceste application generale du mot est un grand vice dans la devise, qui doit estre si particuliere, \& dont les paroles ne doivent estre propres ni convenables, qu'à la personne en faveur de laquelle la devise est inventée» (H. EsTIENNE, L'Art de faire des devises, Paris, Paslé, 1645, pp. 86-87).

(30) Per approfondimenti circa la fortuna seicentesca in ambito francese delle raccolte cartacee e delle gallerie di uomini illustri ispirate all'opera del Giovio, si veda E. POMMIER, Il ritratto. Storia e teorie dal Rinascimento all'Età dei Lumi, traduzione di M. Scolaro, Torino, Einaudi, 2003, pp. 179-194, dove non mancano i riferimenti all'opera di Bussy.

(31) Scrive Bussy ricordando i fatti della primavera del 1656: «Il faut savoir que mon premier dessein (apres celui de m'amuser) a été que mon fils apprît ici mille détails qui coûtent (pour apprendre d'ailleurs) de longues expériences» (Comte de Bussy-Rabutin, Mémoires cit., p. 199).

(32) Tra i contributi inerenti alla pedagogia gesuita e al ruolo centrale delle immagini si segnalano i seguenti saggi: La «Ratio studiorum». Modelli culturali e pratiche educative dei gesuiti in Italia tra Cinque e Seicento, a cura di G.P. Brizzi, Roma, Bulzoni, 1981; R. Dekoninck, Ad imaginem. Statuts, fonctions et usages de l'image dans la littérature spirituelle du XVII siècle, Genève, Droz, 2005; A. SPICA, Les jésuites et l'emblématique, «Dix-septième siècle» 237, ottobre-dicembre 2007, pp. 633-651. Per una bibliografia completa dell'emblematica gesuita si veda G.R. DimLER, Jesuit emblem books. An overview of research past and present, in Emblem studies in honour of Peter M. Daly, edited by M. Bath, P.F. Campa, D.S. Russell, Baden-Baden, Koerner, 2002, pp. 63-122.

(33) Il motto è riferito da Bouhours al «genie sublime de Henri de Bourbon», padre del Grand Condé (D. BOuHOuRs, Les entretiens cit., p. 394). 
il proprio giudizio di quando scriveva l'Histoire amoureuse. Così Madame de La Baume, responsabile della diffusione non autorizzata dell'Histoire, è «la plus jolie maittresse du Royaume et la plus aymable si elle n'eust été la plus infidelle»; più drasticamente la contessa d'Olonne, che nell'Histoire contratta i suoi incontri amorosi e che nemmeno l'amico Saint-Évremond può difendere del tutto ${ }^{34}$, riceve la didascalia più allusiva: «la plus belle femme de son tems, mais moins fameuse pour sa beauté que pour l'usage qu'elle en fit». Inespressiva e impersonale nei ritratti delle altre gallerie, all'interno della Tour dorée la didascalia scivola nella relatività dell'opinione e nella maldicenza personalizzata. Quando poi la notizia delle sottoscrizioni arriva a Parigi la duchessa di Nemours si rifiuta di mandare il suo ritratto per paura, riferisce la Montmorency, che Bussy non parli d'altro che dei suoi amori. Il conte deve desistere, non prima di aver precisato di non avere «une souscription offensante dans trois cents portraits», di aver citato le parole per la contessa d'Olonne come un'eccezione e aver ristabilito il vero sulle sospettose amiche parigine (《j'ai des amies qui ne sont pas des vestales» ${ }^{35}$ ).

Nella sua forma attuale, che tiene conto di interventi successivi ${ }^{36}$, anche la salle de devises ripropone la forma base dell'universo di Bussy: centro immobile attorno a cui ruotano frammenti che lo rendono un po' più reale, il ritratto del conte è circondato dalle sue imprese e dai disegni delle migliori residenze nobiliari di Francia. Sparse su ogni parete, le devises di Bussy non sono originali. Il principio di trasferibilità enunciato da Estienne (l'impresa che mi è già servita potrà servire ad altri «pour exprimer la mesme inclination ${ }^{37}$ ) è alla base dei prontuari simbolici di maggior fortuna, aggregati con criteri variabili: il Mondo simbolico (1669) dell'abate Picinelli classifica le imprese per corpi, tra naturali e artificiali, dai più nobili ai più umili; nelle sue Devises (1654) Boissière impone una trattazione per generi (eroico, amoroso, burlesco, da torneo o carosello) raccolta da Bouhours negli Entretiens.

Gli stessi Entretiens, in particolare il sesto, sono la fonte primaria da cui Bussy trae spunti per le sue decorazioni. Il conte li legge subito, nell'agosto del 1671, quando i lavori nella sua residenza non sono ancora conclusi: tutte le devises del salone principale sono contenute nelle fitte pagine del gesuita, ma è anche probabile che Bussy ne conoscesse già alcune tratte dall'Art de devises (1666) di Le Moyne e magari dal trattato manoscritto di Monsieur Clément, noto allo stesso Bouhours. Dal punto di vista tematico le devises di Bussy ricoprono per intero i principali generi fissati da Boissière. E se alcune potrebbero appartenere a qualsiasi signore di Francia dotato di un grande lignaggio e di onori militari, altre vengono scelte dal conte per una rispondenza più stretta alla propria vicenda: come la meridiana e il diamante più solido che splendente.

Una lumaca appare in primo piano su un'impresa che ha per sfondo il castello di Bussy, sotto un cielo nuvoloso e il cartiglio con il motto italiano «In me me involgo». La devise è illustrata negli Entretiens, ma il motto risale all'estro di Monsieur

(34) Il ritratto della contessa precede di pochi anni l'Histoire amoureuse: «il n'y a rien de si malheureux que de vous aimer; mais rien de si difficile que de ne vous aimer pas» (SAINT-ÉvrEmOND, Caractère de Madame la Comtesse d'Olonne, in ID., Euvres en prose, introduction, notices et notes par R. Ternois, Paris, Didier, 1962, vol. I, p. 24).

(35) Correspondance de Roger de Rabutin cit., vol. I, p. 332.

(36) Gli interventi più considerevoli sulla residenza, tesi a rispettare e a rinforzare i programmi stabiliti dallo stesso Bussy, furono attuati sotto la direzione di Jean-Baptiste César de Sarcus tra il 1835 e il 1854. Capitano di cavalleria ed erudito, il conte è anche l'autore di un volume dedicato alla storia del castello (Comte de Sarcus, Notice historique et descriptive sur le château de Bussy-Rabutin, Dijon, Tricault, 1854). Per un resoconto più dettagliato della storia della residenza, oggi tutelata dal Centre des Monuments Nationaux, si veda J. Kagan, Le château de Bussy-Rabutin, Paris, Editions du patrimoine, 2003.

(37) H. Estienne, L'Art de faire des devises cit., p. 86. 
Clément, che lo usava per un baco da seta che si rinchiude nel suo guscio ${ }^{38}$. Allestita con materia umile, quasi al confine con il burlesco, l'impresa sigilla in realtà il senso dell'esilio di Bussy, la ricerca nella propria identità di un riparo sicuro dai rivolgimenti della fortuna. Il conte vi può fissare uno spunto di saggezza antica ricevuto dall'amico Corbinelli, suo ex ufficiale, che nell'estate del 1669 gli invia un frammento dalle Odi: «Laudo manentem [fortunam]; si celeres quatit I pennas, resigno quae dedit et mea | virtute me involvo» ${ }^{39}$ (III, 29, vv. 53-56). Senza fare cenno ad una futura devise, nella sua risposta di fatto Bussy ne riporta un annuncio, nel momento in cui riconosce qualcosa che lo riguarda e parla per lui: «Horace n'a rien dit qui me touche et qui me plaise si fort, que quand il parle ainsi de la fortune». E poi: «Sans vanité, j'ai dit cela en françois avant que je l'eusse lu dans Horace» ${ }^{40}$.

In altri casi i simboli confermano antichi legami di fedeltà e di devozione, nelle armi e negli amori. Bussy rievoca le figure a lui più care, a partire dal vertice: un sole con il motto «Mas virtud que luz» celebra la gloria di Luigi, a cui allude anche uno sparviero che ghermisce una sua preda con il motto «Non sibi sed domino» ${ }^{41}$. Un cumulo di brace accesa sotto il motto «Splendescam da materiam» ricalca l'impresa del reggimento di Monsieur Le Prince; un razzo sgargiante che si innalza verso il cielo con il motto «Da l'ardore l'ardire» è un tributo al maresciallo Bassompierre ${ }^{42}$. Le devises amorose vanno invece ricondotte, con qualche incertezza nell'attribuzione, alle due mogli di Bussy e alla sua maîtresse, Madame de Montglas, che riceve un piccolo ciclo pittorico a parte, carico di malignità e di rancore: una tortorella poggiata su un ramo con il motto «Piango la sua morte e mia vita» e un cielo stellato privo di luna accompagnato dalle parole «Non mille quod absens» ricordano senza dubbio la prima sposa del conte, Gabrielle de Toulongeon, morta nel $1646^{43}$. La maîtresse infedele, la seconda moglie Louise de Rouville e forse la stessa Sévigné si spartiscono altre imprese, frammenti di un discorso amoroso ancora in corso, reso urgente più che sospeso dall'esilio: due mani intrecciano una corda tesa da un telaio con la frase «Recedendo vincula crescunt», un usignolo canta sulla cima di un albero ( De mi amori me canto»), una mano versa con una brocca un filo d'acqua sulla calce, da cui si leverà un debole fumo per corrispondere al motto «E fredda m'accende» ${ }^{44}$. L'immagine di una

(38) D. BouHOuRs, Les entretiens cit., p. 397.

(39) «La lodo quando resta: ma se batte | le ali veloci, rendo ciò che ha dato I e mi ammantello in ciò che vale in me, I cerco la buona povertà indotata» (Quinto Orazio Flacco, Odi e Epodi, introduzione di A. Traina, traduzione e note di E. Mandruzzato, Milano, Rizzoli, 1985, p. 333).

(40) Correspondance de Roger de Rabutin cit., vol. I, p. 212.

(41) Bouhours riferisce che la prima devise è stata inventata per il re da Monsieur Clément: «il faut avoir un discernement fin \& beaucoup de délicatesse dans l'esprit, pour s'appercevoir que ce bel astre, tout brillant qu'il est, a plus de vertu que d'éclat» (D. Bounours, Les entretiens cit., p. 370). L'impresa viene citata da P. Le Moyne (De l'Art de Devises, avec divers recueils de Devises du mesme Autheur, Paris, Cramoisy, 1666, p. 45). La seconda devise rappresenta «la fidelité d'un General d'armée envers son Prince» (D. BouHOurs, Les entretiens cit., p. 394). Un'altra impresa presenta un riferimento diretto al sovrano: una bandiera sgualcita con il motto italiano «E lacero ogni virtù spira», inventata da Monsieur Clément per i feriti nel servizio del re (ivi, p. 397).

(42) La devise di Bassompierre, nota a Le Moyne, viene riportata anche nella raccolta di Boissière (Les Devises de Monsieur de Boissière cit., p. 18).

(43) Entrambe le imprese vengono citate e spiegate da Bouhours. La prima, già nota a Le Moyne (P. LE Moyne, Devises heroiques et morales, Paris, Courbé, 1649, p. 39), viene riferita a un vedovo (D. BouHOURS, Les entretiens cit., p. 407). La seconda rappresenta la condizione di un uomo lontano dalla persona amata: come le stelle non producono un chiarore pari a quello della luna, così nessuno può sostituire o avvicinare la persona da cui si è allontanato (ivi, p. 407).

(44) La devise con il telaio è attestata solo tra le imprese amorose della raccolta di Boissière (Les Devises de Monsieur de Boissière cit., p. 96). La seconda appare in Bouhours accompagnata da un commento che mantiene incerta l'allusione diretta allo stesso Bussy: «un homme de la Cour qui a beaucoup d'esprit \& de réputation, pour déclarer qu'il ne fait des vers que quand il aime, a peint un Rossignol sur un arbre en 
cipolla, inventata secondo Tesauro dal Cavalier Risentito per una mascherata, parla invece soltanto di Bussy, come una confessione burlesca della sua prontezza nel rendere un colpo ricevuto: «Chi mi morderà piangerà».

Quando deve scegliere la sua devise personale Bussy risale alle ragioni prime della tensione che regola i suoi rapporti con il mondo: ne rivela, attraverso le lettere, il legame insospettabile con una bontà naturale che si è ormai perduta. La destinataria è sempre la Scudéry: «La nature m'avoit fait tendre pour tout le monde; mais le monde m'a endurci pour lui, hors pour mes amis, pour lesquels j'ai ramassé toute ma tendresse ${ }^{45}$. Il motto «Velantur mollia duris» su un castagno carico di frutti è la traduzione simbolica della conclusione di Bussy sulle proprie asprezze e sulla permanenza, in esse, di una certa dolcezza nascosta ${ }^{46}$. Come la devise che il conte dice sua, già portata dallo spietato Urbano VIII (lo sapeva Bussy?), nemico giurato del Pallavicino e degli spiriti libertini:

Il est vrai que je suis né naturellement doux et tendre; aussi ai-je pris pour ma devise une ruche de mouches à miel avec ce mot: Spontè favos, aegrè spicula «la douceur naturelle et l'aigreur étrangère». Mais la pratique du monde, qui la plupart ne vaut rien, m'a donné de l'aigreur aux occasions où il en faut avoir, et il n'y en a point où elle soit mieux employée que sur le sujet de madame de Montglas ${ }^{47}$.

La perfidia verso la sua maîtresse è dunque programmatica, vince la prova della teoria. Dai primi giorni di esilio fino al 1675, quando Bussy la consola per la morte del marito, Cécile-Elisabeth contessa di Cheverny, poi marchesa di Montglas, è la vittima designata su cui il conte infierisce al punto di inquietare la Scudéry, che la frequenta e la dice spesso turbata dal pensiero del vecchio amante. Colpevole di tradimento durante la prigionia alla Bastiglia, dopo un legame più che decennale, Bussy la giudica con rigore marziale e nelle lettere per qualche anno la condanna a una sentimentale abolitio nominis: Cécile sarà solo «l'Infidèle», «l'Inconstante», ma anche «Satan»e «le diable», dedicataria di vari «terribles rondeaux» biasimati dalla Scudéry, oltre che dei ciclo dei «mostri» sui pannelli di Bussy-Le-Grand.

Nella produzione figurativa moderna nessun volto di donna ha ricevuto le attenzioni particolari che il conte riserva a Cécile in questo gruppo di devises. Per il suo bizzarro atelier sentimentale Bussy non può che violare in vari punti la teoria che i trattati tentano a fatica di fissare, contraddicendola in pieno nei contenuti fondamentali. Il conte chiama «mostri» le figure chimeriche che resuscita e fa rappresentare innanzitutto perché il volto di Cécile, ridisegnato dagli eredi per precisarne la fisionomia, si ripete da un pezzo all'altro, finendo sul corpo di una rondine e di una sirena, in uno spicchio di luna, sul piatto di una bilancia e come viso di un'allegoria della Fortuna. Così, Bussy lo sa benissimo, uno dei principi più convalidati dai trattati di imprese è già fuori uso: «Ces devises ne sont pas dans les règles, car il ne doit point y avoir de figures humaines; mais comme les monstres y peuvent entrer, il n'y a qu'à les regarder sous cette idée» ${ }^{48}$.

fleur» (D. BouHours, Les entretiens cit., p. 442). L'impresa della calce è attribuita a Clément (ivi, p. 368) e sarà anche nel secondo volume della Philosopbie des images di Menestrier.

(45) Correspondance de Roger de Rabutin cit., vol. II, p. 59.

(46) Secondo Bouhours, la devise si inserisce invece nella critica ai devoti: è stata inventata per colpire «un faux Devot qui affecte une mine austere, \& qui mene une vie douce» (D. BouHours, Les entretiens cit., p. 403).

(47) Correspondance de Roger de Rabutin cit., vol. II, p. 133. Il riferimento a Urbano VIII, papa dal 1623 al 1644, è segnalato dallo stesso Bouhours.

(48) Ivi, vol. I, p. 71. Nella stessa lettera (28 ottobre 1667) Bussy elenca e descrive brevemente le devises indirizzate alla Montglas. La questione della presenza di una figura umana nel corpo dell'impresa viene 
Al di là della presenza, in tutte, di uno sfondo spesso riconoscibile ma sconsigliato dalla regola, le devises satiriche di Bussy contraddicono di fatto la natura stessa di questa particolare produzione; se si tratta innanzitutto di esprimere un'inclinazione o un disegno nobile, «qualcosa - scriveva Bouhours - di grande e illustre», i fumi acri della satira non possono che restarne fuori. Alludendo, senza fare nomi, anche a Bussy e alla Carte de Braquerie, Le Moyne si domandava perché mai la maldicenza cortigiana, dopo aver già ispirato addirittura le carte geografiche, dovesse anche contaminare le imprese. Lo stesso Bouhours sembra pensare al Rabutin e alla sua sventura presso il re quando dichiara il proprio accordo con l'altro gesuita: «je ne conseillerai jamais à personne de faire des Devises satiriques, non plus que des libelles diffamatoires». Ma alla fine, considerando i tratti dello spirito del suo tempo, Bouhours riusciva a rispondere, lievemente sconsolato, anche all'incredulità di Le Moyne: forse le imprese satiriche si moltiplicheranno, «car la raillerie \& la médisance regnent plus que jamais dans le monde» ${ }^{49}$.

Estraneo a una derisione improvvisata e saltuaria, Bussy produce la sua piccola galleria senza rinunciare a riferimenti alti, piegati verso la bassezza morale che riconosce nella sua infedele. La rappresentazione più ricercata della colpa di Cécile non deriva neppure dall'ambito specifico della devise, ma da una classica maschera allegorica, tra le più popolari nella precedente produzione emblematica francese e nel repertorio dell'Iconologia del Ripa che Baudoin traduceva e pubblicava nel 1644: nei disegni che l'erudito borgognone Pierre-Louis Baudot abbozza nel 1781 durante una visita al castello, prima delle modifiche ottocentesche, Cécile è in piedi su una sfera dotata di ali, regge nella destra una corona d'alloro e nella sinistra una piccola canna appoggiata al petto, mentre il vento agita un panno della sua veste. Più che alla Fortuna di Alciato e Corrozet ${ }^{50}$, la devise di Bussy guarda all'allegoria dell'Instabilità o Incostanza nell'Iconologia del Ripa:

Donna vestita di molti colori, con la man destra s'appoggi a una canna con le foglie, e sotto i piedi tenga una palla. Vestesi di varii colori l'instabilità, per la frequente mutation di pensieri dell'uomo instabile. Si appoggia ad una fragil canna sopra alla palla, percioché non è stato di condizione alcuna dove la volubil mente fermandosi si assicuri, e dove non si appigli conforme alle cose più mobili e meno certe $\mathrm{e}^{51}$.

Il motto della devise meno regolare dell'intera serie accresce l'identificazione della donna con l'allegoria, sua pari nell'imperfezione e nelle debolezze: «Ambo leves, ambo ingratae». Nell'ora del tradimento e negli anni successivi il conte è occupato ad elaborare una sola ossessione sentimentale, come un vero libertino non farebbe mai. Di fatto, l'improvvisa metamorfosi di Cécile in una creatura frivola e ingrata è veramente l'evento decisivo nella sua storia sentimentale. «Ma fortune que je voyois

affrontata con soluzioni alterne almeno fino a Bargagli, che fissa una regola rigida di esclusione totale che avrà poi fortuna anche in Francia. La ragione primaria per l'esclusione del corpo umano e delle sue parti verrà ripresa alla lettera, tra gli altri, anche da Le Moyne e da Bouhours, trovando maggiori resistenze in altri autori, come in Tesauro: se l'impresa si fonda sulla similitudine tra l'uomo che la inventa e l'oggetto rappresentato, il senso dell'operazione decade se l'autore si paragona a un altro uomo («l'huomo cioè, non può dall'huomo propia comparazione ritrarre, o similitudine: dovendosi questa propiamente solo, o da cose levare fra loro di genere, o di spezie diverse», S. BARGAGLI, Dell'imprese cit., p. 49).

(49) D. Bouhours, Les entretiens cit., p. 380.

(50) I riferimenti principali rimandano all'emblema «Ars naturam adiuvans» nella seconda serie degli Emblemi dell'Alciato (Venezia, 1546) e a «L'ymage de la fortune» nell'Hecatomgraphie di Corrozet. La lettura più approfondita dell'emblema dell'Alciato viene offerta da Jean Baudoin nel suo Recueil (J. BAUDOIN, Recueil d'emblemes divers avec des discours moraux, philosophiques et politiques, Paris, Villery, 1639, vol. II, pp. 17-23).

(51) C. RIPA, Iconologia, a cura di S. Maffei, testo stabilito da P. Procaccioli, Torino, Einaudi, 2012 [ristampa di: Roma, Lepido Facii, 1603], p. 290. 
traversée de mille ennemis, me tenoit en de continuelles alarmes, et je n'y aurois pu résister - scrive il conte nel 1668 alla contessa di Fiesque - sans la belle passion que vous connoissiez, dont je faisois alors tous mes plaisirs ${ }^{52}$. Come la saggezza per un filosofo antico, per Bussy la sola costanza amorosa avrebbe potuto qualcosa contro i colpi della sventura, secondo una delle Maximes che piacevano tanto al re, carica per il suo autore di cattivi presagi (la prigione, l'esilio):

Tant qu'un amant fort amoureux

Est sûr du cœur de sa maitresse,

La fortune la plus traitresse,

Ne le peut rendre malheureux.

Sa prison ne sauroit ébranler sa costance,

Il la sent aussi peu que s'il étoit brutal:

Et même son exil ne lui paroît un mal

Que parce qu'il est une absence ${ }^{53}$.

Quando la costanza di Cécile, souverain bien, svanisce del tutto, la rovina dell'ideale amoroso di Bussy è fragorosa e irreparabile. La sua carriera libertina si chiude su un'articolata e insistente invettiva anche perché, a partire dalla realtà, la donna compie un percorso perfetto, altamente produttivo sul piano simbolico. Da forze opposte e in equilibrio fra loro, l'amante e la Fortuna si allineano e hanno un solo volto:

La fortune et l'amour m'accablaient d'inquiétude; mais ce qui augmentait celle de l'amour, c'est que je m'étais toujours défié de la fortune et jamais de ma mâtresse. Tout ce que je puis dire en faveur de celle-ci, c'est que je crois qu'elle ne m'eût point quitté, si l'autre (la fortune) ne lui en eût montré le chemin ${ }^{54}$.

Sorta di manifesto sentimentale, la devise della Fortuna è il cuore di un ciclo amoroso di cui le altre sono focalizzazioni particolari sulle qualità che questa include: incostanza, leggerezza, falsità. Il volto di Cécile sorvola acque agitate su un corpo di rondine che la grossolana diceria cortigiana trasformerà poi in pipistrello ${ }^{55}$, privando il tutto di senso: con il motto «Fugit Hyemes» solo il piccolo animale è adatto a raffigurare, precisa Menestrier, «ces amis infideles qui abandonnent leurs amis dans les mauvais succez de leurs affaires $»^{56}$. Il tema dell'incostanza vuole invece il volto della marchesa incastonata in uno spicchio di luna sotto le parole «Haec ut illa», secondo una lettura canonica che lo stesso Baudoin avanza nella sua riscrittura del Ripa, per giustificare l'allegoria dell'incostanza come donna che regge una piccola luna, «le plus muable des Astres»: «d'où vient qu'il est dit dans l'Escriture, Que l'insensé change comme elle, \& qu'il ne demeure jamais en un mesme estat» ${ }^{57}$. Creatura seducente

(52) Correspondance de Roger de Rabutin cit., vol. I, p. 82.

(53) Mémoires de Roger de Rabutin, comte de Bussy cit., vol. II p. 190.

(54) Comte de Bussy-Rabutin, Mémoires cit., p. 329.

(55) Scrive così Bussy alla Montpensier ancora nel 1689, dopo aver chiarito la confusione tra pipistrello e rondine: «Pour mille raisons, je voudrois bien que l'hirondelle eût passé la mer cinq ou six ans plus tôt qu'elle ne fît; je vois bien ce qui l'en empêcha: c'est que les beaux jours n'étoient pas encore passés alors» (Correspondance de Roger de Rabutin cit., vol. VI, pp. 253-254).

(56) Il commento di Menestrier riguarda però il motto «Hieme avolant» (C.F. MENESTRIER, La Philosophie des images cit., vol. II, p. 383).

(57) Lo spunto è un'integrazione di Baudoin non presente nel testo del Ripa (C. RIPA, Iconologie, ou Explication Nouvelle de Plusieurs Images, Paris, Guillemont, 1643, vol. II, p. 93). Il riferimento biblico riprende un passo dell'Ecclesiaste: «Homo sanctus in sapientia manet sicut sol: nam stultus sicut luna mutatur» (Eccle. XXVII, 12). 
e ingannevole, Cécile si trasforma anche in una sirena («Allicit ut perdat»), figura che Ripa inseriva nell'allegoria del Piacere nella logica di una fatale deriva degli amanti inebriati, perfetta per Bussy: «il piacere con l'apparente dolcezza mondana manda in ruina i suoi seguaci» ${ }^{58}$. Quando poi fa disegnare il volto della Montglas sul piatto di una bilancia, vinto dal peso di un piatto vuoto («Levior aura»), il conte ci dice che quello spirito volage non ha un vero termine di paragone, compreso l'impalpabile, l'immateriale, il puro nulla. Bussy estremizza qui uno spunto derivato con ogni probabilità da La Perrière, che nel Theatre des bons engins (1544) aveva adottato lo stesso strumento per il corpo dell'emblema della falsa amicizia: una fanciulla regge una bilancia su cui il peso di due mani che si stringono è facilmente superato da una semplice piuma.

In un caso il volto della donna scompare del tutto dalla devise per lasciare spazio a un arcobaleno che si inarca sulla sua residenza sotto il motto «Minus Iris quam mea». Iris, personificazione appunto dell'arcobaleno, è il nome di Cécile prima e dopo l'esilio, nelle Maximes come in tanti componimenti satirici, e un travestimento mitologico attestato nella ritrattistica coeva. Per Bussy i riferimenti tendono ancora una volta all'esaltazione di un vizio e sono l'ennesima rappresentazione dell'illusione di cui Cécile è maestra e padrona. La mescolanza dei colori è già in Ripa un tratto distintivo dell'Instabilità, segno inconfondibile di una «frequente mutation di pensieri». Ma lo spunto decisivo per la rappresentazione della Montglas si trova, con ogni probabilità, nella pagina che nella sua Art de devises Le Moyne dedicava alla simbologia dell'iris, fiore di una «bellezza senza impostura», opposto allo spettacolo sgargiante dell'arcobaleno, pura «apparenza e finzione»:

L'Iris qui se forme dans la nuë est de ce rang-là, elle n'a pas le corps qu'on luy voit; elle n'en à pas mesme les vrayes couleurs. Et comme si ce ne luy estoit pas assez de tromper, elle est encore bizarre \& changeante en ses tromperies ${ }^{59}$.

Come agli occhi di Bussy è ormai diventata la sua amata Cécile.

DANIELE SANTERO

(58) C. RIPA, Iconologia cit., p. 472.

(59) P. Le Moyne, De l'Art de Devises cit., p. 400. 